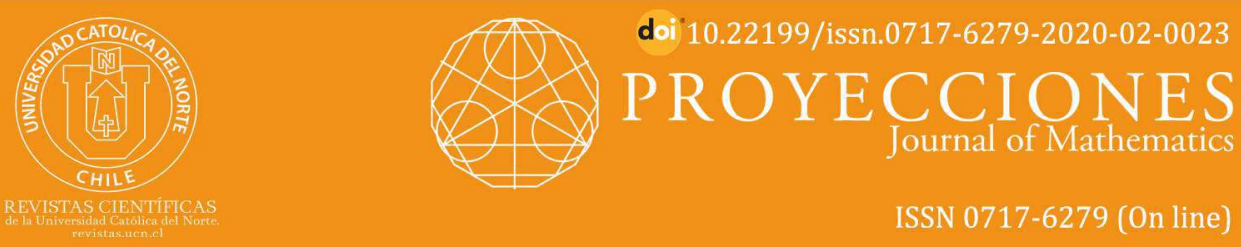

\title{
Global dynamics of COPD and Tuberculosis among smokers
}

\author{
Nita H. Shah ${ }^{1}$ (D) orcid.org/0000-0003-1605-4778 \\ Purvi M. Pandya ${ }^{2}$ (i) orcid.org/0000-0003-1889-093X \\ Moksha H. Satia ${ }^{3}$ (D) orcid.org/0000-0001-6301-9241 \\ Ankush H. Suthar ${ }^{4}$ (D) orcid.org/0000-0001-7583-450X
}

Gujarat University, Dept. of Mathematics, Ahmedabad, GJ, India.

1 nitahshah@gmail.com; ${ }^{2}$ pandya091@gmail.com;

3-mokshasatia.05@gmail.com ; 4@ankush.suthar1070@gmail.com

Received: April 2019 | Accepted: November 2019

\begin{abstract}
:
Chronic Obstructive Pulmonary Disease (COPD) and tuberculosis among smokers have turned out to be major public health concern, accounting for millions of deaths annually. Smoking is becoming like a trend in human society. This adds in maximizing the risk of people suffering from most dreadful health issues and the reason for extensive morbidity and mortality around the world. People who smoke have approximately twice the risk of suffering from tuberculosis and also COPD patients with the history of tuberculosis writhes in agony. To scrutinize spread of this disease, an understanding of the epidemiological relationship between them are important. The relation between COPD, tuberculosis among smokers and medication is studied in this research work and the formulation for the mathematical model is done using system of nonlinear ordinary differential equations. The threshold for smokers suffering from COPD and tuberculosis who needs medication is computed using next generation matrix method. Also, stability analysis and numerical simulations are carried out to validate the data.
\end{abstract}

Keywords: Mathematical model; Basic reproduction number; Stability.

Cite this article as (IEEE citation style):

N. H. Shah, P. M. Pandya, M. H. Satia and A. H. Suthar, "Global dynamics of COPD and Tuberculosis among smokers", Proyecciones (Antofagasta, On line), vol. 39, no. 2, pp. 383-399, Apr. 2020, doi: 10.22199/issn.0717-6279-2020-02-0023.

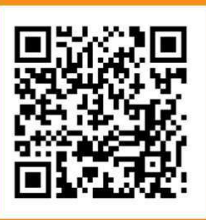

Article copyright: (C) 2020 Nita H. Shah, Purvi M. Pandya, Moksha H. Satia and Ankush H. Suthar. This is an open access article distributed under the terms of the Creative Commons Licence, which permits unrestricted use and distribution provided the original author and source are credited.

(cc) BY 


\section{Introduction}

Smoking is considered to be an alarmingly common issue in the society. There are different modes through which people inhale these harmful smokes. It can be in the form of bidis, cigarette, cigar, e-cigarette, hukkah and many more [10]. Among youths, vaping or juuling is usually found which may lead to smoke addiction [2]. Though the modes of smoking are different but each harms the body in the same manner. The reason to consume this poisonous smoke varies person to person, it may be for enjoyment, stress relief, weight control, peer pressure, etc. In some cases, people smoke as it is also considered as a status symbol. Due to which many horrific diseases prevail in one's body. It is also observed that not only smokers but people around them are also affected. So, individuals are affected directly as well as indirectly due to smoking. It mostly affects lungs which disturbs breathing functions. In the beginning it is in the form of coughs, colds, wheezing and asthma but sometimes it leads to pneumonia, emphysema and lung cancer $[1,18]$. Also, maximum cases of tuberculosis and COPD are observed. Smoke mainly damages the airways, air sacs and the lining of the lungs. So, in case of COPD, the progression of the condition can only be slowed down by treatment as it permanently damages the lungs. While tuberculosis can be cured if prescribed course is completed with proper precautions. People do smoke despite of being aware of these outcomes. It's very difficult for a person mentally as well as physically to stop this addiction. Science has progressed to a great level to stop them from this urge of smoking. Medication in the form of nicotine chewing gum, nicotine patch, nicotine spray, nicotine nasal etc. are used to quit smoking $[4,8]$.

A lot of related study is done which gives the idea about issues and problems prevailing in the society due to smoking. Davies et al. [5] reviewed an epidemiological relation between smoking and tuberculosis from the UK, India, china and the USA. Also, Zaher et al. [19] assessed the burden of illness like COPD, CHD and lung diseases due to smoking. A populationbased study was done by Buist et al. [3] which aimed to measure the international variation in the prevalence of COPD by age, sex, and smoking status. Lin et al. [14] studied a time-based, multiple risk factor and modelling to show the effects of smoking and solid-fuel use on COPD, lung cancer and tuberculosis in china. Also, Salvi and Barnes [16] discussed about chronic obstructive pulmonary disease found in non-smokers. While Lee et al. [12] analyzed a nationwide cohort study in Taiwan about the 
risk factors for pulmonary tuberculosis in patients with chronic obstructive airway disease. Also, whether age matters on the impact of cigarette smoking on tuberculosis was discussed by Feng et al. [7]. Moreover, Jha et al. [9] did a nationally representative case-control study of smoking and death in India. Even the colliding epidemics of tuberculosis, tobacco smoking, HIV and COPD were analyzed by van Zyl Smit et al. [17] which are the global lung health issues. Leung et al. [13] investigated about the relation between smoking and tuberculosis and also how it adversely affects treatment response, outcome and relapse in tuberculosis.

The mathematical model for COPD and tuberculosis among smokers is designed and formulated in section 2. Stability at all equilibrium points is analyzed in section 3 followed by numerical simulation in section 4 , then finally concluding the model in section 5 .

\section{Mathematical Model}

In this mathematical model, the transmission of COPD and tuberculosis among smokers is studied. It is based on SIRS-model. This model as shown in Figure 1 comprises of four compartments, namely, smokers $\left(S_{M}\right)$ who are likely to get infected by COPD $\left(I_{1}\right)$ and tuberculosis $\left(I_{2}\right)$. In some cases, individuals suffering from COPD may suffer from tuberculosis and viceversa. To get cured or quit smoking they procure medication $(M)$. After getting cured, they may again start smoking. 
Table 1: Model parameters and their interpretation

\begin{tabular}{clc}
\hline Notation & Description & Parametric values \\
\hline$B$ & Birth Rate & 0.4 \\
\hline$\alpha_{1}$ & $\begin{array}{l}\text { Rate at which individuals suffers from COPD } \\
\text { due to smoking }\end{array}$ & 0.4 \\
\hline$\alpha_{2}$ & Rate at which smokers get TB infection & 0.3 \\
\hline$\alpha_{3}$ & Rate of TB patients anffering from COPD & 0.75 \\
\hline$\alpha_{4}$ & Rate of COPD patients suffering from TB & 0.4 \\
\hline$\alpha_{5}$ & $\begin{array}{l}\text { Rate at which COPD patients starts medica- } \\
\text { tion }\end{array}$ & 0.4 \\
\hline$\alpha_{6}$ & $\begin{array}{l}\text { Rate at which TB patients procure medica- } \\
\text { tion }\end{array}$ & 0.8 \\
\hline$\alpha_{7}$ & $\begin{array}{l}\text { Rate of individuals after getting cured again } \\
\text { starts smoking }\end{array}$ & 0.2 \\
\hline$\mu_{d}$ & Death rate due to disease & 0.3 \\
\hline$\mu$ & Natural Death rate & 0.1 \\
\hline
\end{tabular}

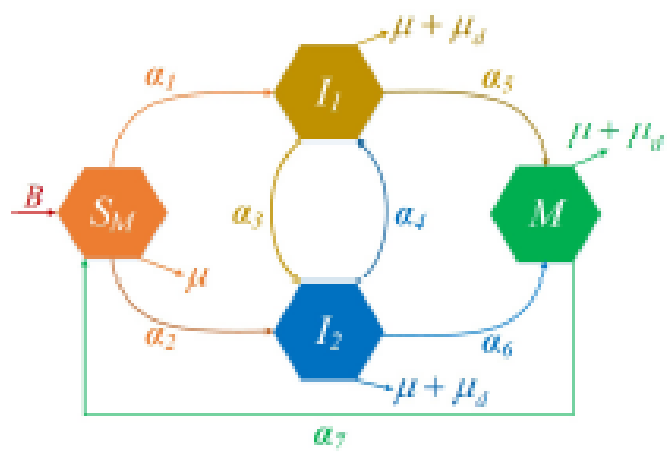

Figure 1: Mathematical model 
Using parameters, the mathematical model is formulated. The system of non-linear differential equations is as follows:

$$
\begin{aligned}
\frac{d S_{M}}{d t} & =B-\alpha_{1} S_{M} I_{1}-\alpha_{2} S_{M} I_{2}+\alpha_{7} M-\mu S_{M} \\
\frac{d I_{1}}{d t} & =\alpha_{1} S_{M} I_{1}-\alpha_{3} I_{1}+\alpha_{4} I_{2}-\alpha_{5} I_{1}-\left(\mu+\mu_{d}\right) I_{1} \\
\frac{d I_{2}}{d t} & =\alpha_{2} S_{M} I_{2}+\alpha_{3} I_{1}-\alpha_{4} I_{2}-\alpha_{6} I_{2}-\left(\mu+\mu_{d}\right) I_{2} \\
\frac{d M}{d t} & =\alpha_{5} I_{1}+\alpha_{6} I_{2}-\alpha_{7} M-\left(\mu+\mu_{d}\right) M
\end{aligned}
$$

where, $S_{M}+I_{1}+I_{2}+M \leq N$. Also, $S_{M}>0 ; I_{1}, I_{2}, M \geq 0$.

Adding the above differential equations of system (1.1), we have $\frac{d}{d t}\left(S_{M}+I_{1}+I_{2}+M\right)=B-\mu\left(S_{M}+I_{1}+I_{2}+M\right)-\mu_{d}\left(I_{1}+I_{2}+M\right) \geq 0$ which implies that $\lim _{t \rightarrow \infty} \sup \left(S_{M}+I_{1}+I_{2}+M\right) \leq \frac{B}{\mu}$.

Therefore, the feasible region of the model is $\Lambda=\left\{\left(S_{M}, I_{1}, I_{2}, M\right) \in R^{4}: S_{M}+I_{1}+I_{2}+M \leq \frac{B}{\mu}\right\}$. Now, the diseasefree equilibrium point of the model is $Y_{0}=\left(\frac{B}{\mu}, 0,0,0\right)$ and endemic point is $Y^{*}=\left(S_{M^{*}}, I_{1}{ }^{*}, I_{2}{ }^{*}, M^{*}\right)$

where,

$$
I_{1}^{*}=\frac{S_{M^{*}=r}(B-\mu r)\left(\alpha_{7}+\mu+\mu_{d}\right)\left(\alpha_{1}\left(\alpha_{4}+\alpha_{6}-\alpha_{2} r+\mu+\mu_{d}\right)-\alpha_{2} \alpha_{4}\right)}{r \alpha_{1} \alpha_{2}\left(\alpha_{6}-\alpha_{5}\right)\left(\mu+\mu_{d}\right)},
$$




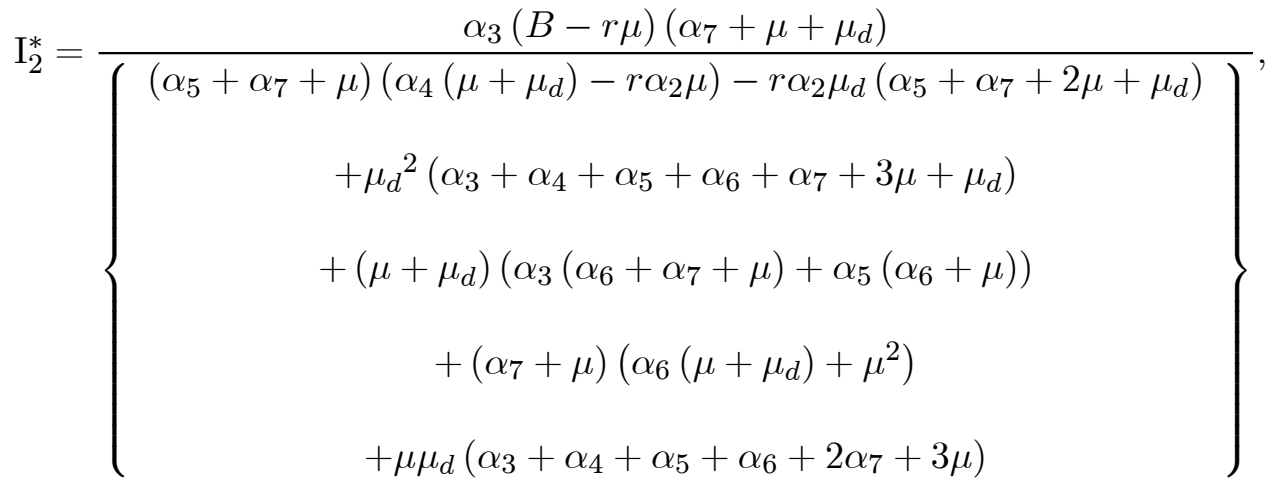

$$
\begin{aligned}
& M^{*}=-\left[\left(\alpha_{1}-\alpha_{2}\right) \mu_{d}^{2}\left(\alpha_{5}\left(\mu_{d}-\alpha_{2} r\right)+\alpha_{1} \alpha_{3} \alpha_{6} \mu r\right)-\mu_{d} \alpha_{1} \alpha_{3}\left(\alpha_{5}+\alpha_{6}\right)\right. \\
& \left(B \mu_{d}\left(\alpha_{3}-\alpha_{2}\right)+\alpha_{2} \mu r\left(\alpha_{3}+\alpha_{4}\right)\right)+\left(\alpha_{7}+2 \mu\right)\left\{( \alpha _ { 6 } + \mu ) \left(\alpha_{1} \alpha_{2} \alpha_{5}^{2}\right.\right. \\
& \left.(B-\mu r)-\alpha_{5}^{2} \mu\left(\alpha_{1}\left(\alpha_{6}+\mu\right)-2 \alpha_{2} \alpha_{3}\right)\right)+\mu_{d} \alpha_{5} \alpha_{6}\left(\alpha_{1}\left(B \alpha_{2}+\alpha_{6} \mu\right)\right. \\
& \left.+\mu\left(2 \alpha_{1} \alpha_{4}-\alpha_{2}\left(2 \alpha_{3}+\alpha_{4}\right)\right)\right)+\alpha_{5} \mu^{2}\left(3 \mu_{d}^{2}\left(\alpha_{1}-\alpha_{2}\right)+\alpha_{1} \alpha_{5}\left(\alpha_{3}+2 \alpha_{4}\right)\right) \\
& -\alpha_{2} \alpha_{6} \mu\left(\alpha_{1} \mu_{d} r\left(\alpha_{3}+\alpha_{5}\right)+\alpha_{5}\left(\alpha_{4} \alpha_{5}+2 \alpha_{1} \alpha_{3} r\right)\right)+\mu_{d} B \alpha_{1} \alpha_{5} \\
& \left.\left(\alpha_{2}\left(\alpha_{3}+\alpha_{4}\right)-\alpha_{1} \alpha_{6}\right)\right\}+\left(2 \alpha_{7}+3 \mu\right)\left\{-\mu_{d} \alpha_{5} \mu\left(\alpha_{1}-\alpha_{2}\right)\left(B \alpha_{1}+\alpha_{2} \mu r\right)\right. \\
& +\alpha_{5} \mu^{2}\left(\alpha_{2}\left(\alpha_{2} \alpha_{5} r-\alpha_{6}\left(\alpha_{5}+\mu_{d}\right)-\left(\alpha_{5} \mu+2 \mu_{d} \alpha_{3}\right)\right)+2 \mu_{d}\left(\alpha_{1}\left(\alpha_{3}+\alpha_{6}\right)\right.\right. \\
& \left.\left.\left.+\alpha_{4}\left(\alpha_{1}+\alpha_{2}\right)\right)\right)\right\}+\left(\alpha_{5}+\alpha_{7}+\mu\right)\left\{B \alpha _ { 1 } \alpha _ { 5 } \left(\alpha _ { 2 } r \left(\alpha_{4}\left(\alpha_{1}+\alpha_{2}\right)\right.\right.\right. \\
& \left.\left.-\alpha_{2} \alpha_{3}\right)-\alpha_{4}^{2}\left(\alpha_{1}-\alpha_{2}\right)\right)+\alpha_{2} \alpha_{5} \mu\left(\alpha_{2} r\left(\alpha_{3}\left(\alpha_{3}+\alpha_{4}\right)+\left(\mu_{d}+\alpha_{5}\right)^{2}\right)\right. \\
& \left.\left.-\mu_{d} \alpha_{4}\left(\mu_{d}+\alpha_{3}\right)\right)\right\}+\left(\alpha_{6}+\mu\right)\left\{\alpha_{1} \alpha_{5}^{2}\left(B \alpha_{1}-\alpha_{5} \mu\right)\left(\alpha_{2} r-2 \mu_{d}-2 \alpha_{4}\right)\right. \\
& \left.+\alpha_{2} \alpha_{5}{ }^{2}\left(\alpha_{3}+\alpha_{5}\right)\left(B \alpha_{1}-\mu\left(\alpha_{3}+\alpha_{5}\right)\right)-\alpha_{1} \alpha_{3} \alpha_{6}{ }^{2} \mu\left(\alpha_{3}+\alpha_{5}\right)\right\}+\left(\alpha_{7}\right. \\
& +\mu)\left\{( \alpha _ { 6 } + \mu ) \left(\alpha_{1} \alpha_{5} \mu\left(B \alpha_{2}+\mu\left(\alpha_{6}+\mu\right)\right)-B \alpha_{1}^{2} \alpha_{5}\left(2 \alpha_{4}+\alpha_{6}+\mu\right)\right.\right. \\
& \left.-\alpha_{2} \alpha_{5} \mu\left(\alpha_{3}+\mu^{2}\right)\right)-\alpha_{1}\left(\alpha_{4}+\alpha_{6}+\mu\right)\left(\alpha_{2} \alpha_{5} \mu^{2} r+B \alpha_{1} \alpha_{3} \alpha_{6}\right) \\
& +\alpha_{1} r\left(B \alpha_{2} \alpha_{5}\left(\alpha_{1} \alpha_{6}+\mu\left(\alpha_{1}-\alpha_{2}\right)\right)+\alpha_{3} \alpha_{6} \mu\left(\alpha_{1} \mu-\alpha_{2}\left(\alpha_{3}+\alpha_{4}\right)\right)\right) \\
& +\alpha_{2} \mu^{2} r\left(\alpha_{2} \alpha_{5}\left(2 \alpha_{3}+\alpha_{4}+\mu\right)-\alpha_{1} \alpha_{3}\right)+B \alpha_{1} \alpha_{2}\left(\alpha_{4} \alpha_{6}\left(\alpha_{3}+\alpha_{5}\right)+\alpha_{5} \mu\right. \\
& \left.\left(\alpha_{3}+2 \alpha_{4}\right)\right)+\alpha_{5} \mu\left(\alpha _ { 1 } \left(\alpha_{3} \mu\left(\alpha_{3}+2 \alpha_{4}+2 \mu\right)+\alpha_{4}\left(\mu\left(\alpha_{4}+2 \mu\right)+2 \alpha_{6}\left(\alpha_{5}\right.\right.\right.\right. \\
& \left.\left.+\mu)))-\alpha_{2} \alpha_{4}\left(\alpha_{3}\left(\alpha_{6}+2 \mu\right)+\mu\left(\alpha_{4}+\alpha_{6}+2 \mu\right)\right)\right)\right\}+\left(\alpha_{5}+\alpha_{7}+2 \mu\right) \\
& \left\{\alpha_{5}{ }^{2}\left(\alpha_{2} r\left(\alpha_{2} \mu\left(2 \alpha_{3}+\alpha_{4}\right)-\alpha_{1}\left(B \alpha_{2}+\alpha_{4} \mu\right)\right)+\alpha_{4}{ }^{2} \mu\left(\alpha_{1}-\alpha_{2}\right)\right)\right\} \\
& +\alpha_{1} \alpha_{3} \alpha_{6}\left(\alpha_{6}+\alpha_{7}+\mu\right)\left\{\alpha_{1} \mu r\left(\alpha_{3}+\alpha_{4}+\alpha_{6}\right)+B\left(\alpha_{2} \mu-\alpha_{3}\left(\alpha_{1}-\alpha_{2}\right)\right)\right\} \\
& +\alpha_{5} \mu\left(\alpha_{7}+3 \mu\right)\left\{\mu_{d}\left(2 \alpha_{1} \alpha_{5} \mu-\alpha_{2} \alpha_{6}\left(\mu_{d}+2 \alpha_{5}\right)\right)-\alpha_{2} \alpha_{5}^{2}\left(\alpha_{6}+\mu\right)\right\} \\
& +\alpha_{1} \alpha_{3} \alpha_{5}\left(2 \alpha_{6}+\alpha_{7}+\mu\right)\left\{\alpha_{2} r\left(B \alpha_{1}-\mu\left(\alpha_{3}+\alpha_{4}\right)\right)-B \alpha_{4}\left(\alpha_{1}-\alpha_{2}\right)\right\} \\
& +\left(\alpha_{3}+\alpha_{4}\right)\left\{\left(\alpha_{1}-\alpha_{2}\right)\left(\mu_{d} \alpha_{5}\left(B \alpha_{1} \alpha_{2} r+2 \mu_{d}^{2} \mu\right)-\mu_{d} B \alpha_{1} \alpha_{3} \alpha_{6}\right)+\mu_{d} \mu\right. \\
& \left.\left(\alpha_{3} r\left(\alpha_{1}^{2} \alpha_{6}+\alpha_{2}^{2} \alpha_{5}\right)-\alpha_{1} \alpha_{3} \alpha_{6}^{2}\right)\right\}+\mu_{d} \alpha_{5} \mu\left(2 \alpha_{5}+\alpha_{7}+2 \mu\right)\left\{\alpha _ { 2 } \left(\alpha_{2} \mu\right.\right. \\
& \left.\left.\left(2 \alpha_{3}+\alpha_{4}-\alpha_{1}\left(B \alpha_{2}+\alpha_{4} \mu\right)\right)+\alpha_{4}^{2}\left(\alpha_{1}-\alpha_{2}\right)\right)\right\}+\alpha_{5} \mu^{2}\left(\alpha_{5}+3 \alpha_{7}+5 \mu\right) \\
& \left(\alpha_{1} \alpha_{3} \alpha_{6}-\alpha_{2} \alpha_{4} \alpha_{5}\right)+\alpha_{1} \alpha_{5}\left(2 \alpha_{6}+\alpha_{7}+3 \mu\right)\left(\alpha_{3}^{2} \alpha_{6} \mu-B \alpha_{1}\left(\mu_{d}^{2}+\alpha_{3} \alpha_{6}\right)\right) \\
& +\alpha_{2} \alpha_{4} \alpha_{5}^{2}\left(\alpha_{5}+\alpha_{6}+\alpha_{7}+3 \mu\right)\left(B \alpha_{1}-\mu\left(\alpha_{3}+\alpha_{5}\right)\right)+\alpha_{1} \alpha_{5}{ }^{2}\left(\alpha_{6}+\mu\right)^{2} \\
& \left(\alpha_{5} \mu-B \alpha_{1}\right)+\alpha_{1} \alpha_{3}\left(\alpha_{5}^{2} \mu\left(\alpha_{4}+\alpha_{6}\right)\left(3 \alpha_{6}+\alpha_{7}+2 \mu\right)-\alpha_{5} \alpha_{6} \mu\left(\alpha_{4}+\alpha_{6}\right)\right. \\
& \left.\left(\alpha_{6}-\alpha_{7}-2 \mu\right)+\mu\left(\alpha_{4} \alpha_{6} \mu\left(\alpha_{5}-\alpha_{6}\right)+2 \alpha_{5}\left(\alpha_{5} \alpha_{6} \mu-\mu_{d}^{2} \alpha_{2} r\right)\right)\right)-\mu_{d} \mu
\end{aligned}
$$




$$
\begin{aligned}
& \left(\alpha_{2} \alpha_{5} \mu\left(\alpha_{7}+\mu\right)\left(4 \alpha_{5}+3 \mu\right)-\mu_{d} \alpha_{1} \alpha_{3} \alpha_{6}{ }^{2}-\alpha_{2} \alpha_{5} \mu^{2}\left(5 \alpha_{5}+\mu\right)\right)+\left(\alpha_{6}\right. \\
& \left.+\alpha_{7}+2 \mu\right)\left\{\mu_{d} \alpha_{2} \alpha_{5}\left(B \alpha_{1} \mu_{d}-\alpha_{5}^{2} \mu-2 \mu_{d} \alpha_{3} \mu\right)+B \alpha_{1}\left(\alpha _ { 1 } \alpha _ { 5 } \left(\mu_{d} \alpha_{2} r-\alpha_{3}\right.\right.\right. \\
& \left.\mu)+\alpha_{2} \alpha_{3} \alpha_{6}\left(\alpha_{5}+\mu_{d}\right)\right)+\mu_{d} \alpha_{5} \mu\left(\alpha _ { 1 } \left(\mu_{d} \alpha_{6}-\alpha_{2} \alpha_{5} r+2 \alpha_{5}\left(\alpha_{4}+\alpha_{6}\right)-B\right.\right. \\
& \left.\left.\left(2 \alpha_{4}+\alpha_{6}\right)\right)+\mu_{d}^{2}\left(\alpha_{1}-\alpha_{2}\right)-\alpha_{2}\left(\alpha_{3}\left(\alpha_{3}+2 \alpha_{5}\right)+\alpha_{4}\left(\alpha_{3}+\alpha_{5}\right)\right)\right)+\alpha_{1} \alpha_{3} \\
& \left(\mu_{d} \alpha_{1} \alpha_{6}(\mu r-B)-\alpha_{2} \alpha_{5} \mu r\left(2 \mu_{d}+\alpha_{5}\right)\right)+\mu_{d} \alpha_{5}\left(\alpha _ { 1 } \mu ( \mu _ { d } + \alpha _ { 3 } ) \left(\alpha_{3}+2 \alpha_{4}\right.\right. \\
& \left.\left.\left.+\alpha_{5}\right)+\alpha_{2}\left(\alpha_{4}+\alpha_{5}\right)\left(B \alpha_{1}-\mu_{d}\right)\right)\right\}+\left(\alpha_{5}+\alpha_{6}+2 \mu\right)\left\{\mu _ { d } \alpha _ { 5 } \left(\mu \left(\mu_{d} \alpha_{1}\right.\right.\right. \\
& \left.\left(\alpha_{5}+\mu_{d}\right)+2\left(\alpha_{1} \alpha_{3}-\mu_{d} \alpha_{5}\right)-\alpha_{2} \alpha_{5}\left(2 \alpha_{3}+\alpha_{4}+\alpha_{5}+\alpha_{1} r\right)\right)+\alpha_{1} \alpha_{5} \\
& \left.\left.\left(B \alpha_{2}+2 \alpha_{4} \mu\right)\right)\right\}+\mu_{d}^{2} \alpha_{5}\left(\alpha_{4}+2 \alpha_{5}+\mu\right)\left(\alpha_{4}+2 \alpha_{5}+\mu\right)\left(\alpha_{1} \alpha_{2}\left(B-\alpha_{2} r\right)\right. \\
& \left.+\mu\left(\alpha_{1} \alpha_{4}-\alpha_{2} \alpha_{3}\right)\right)+\mu_{d} \alpha_{1} \alpha_{5} \mu\left(\alpha_{3}\left(\alpha_{5}+\alpha_{6}\right)\left(\alpha_{4}-2 \alpha_{2} r\right)+\left(3 \alpha_{7}+4 \mu\right)\right. \\
& \left.\left(\alpha_{3} \alpha_{6}+\mu^{2}\right)\right)+\mu_{d} \alpha_{5}\left\{\alpha _ { 5 } \mu \left(\left(\alpha_{6}+\mu\right)\left(2 \mu_{d} \alpha_{1}-\alpha_{2} \alpha_{5}\right)+\mu_{d}\left(\mu_{d} \alpha_{1}-\alpha_{2}\left(\alpha_{5}\right.\right.\right.\right. \\
& \left.\left.\left.+\alpha_{7}+3 \mu\right)\right)-\alpha_{2}\left(\alpha_{5} \mu+\alpha_{4}\left(2 \alpha_{7}+7 \mu\right)\right)\right)+B \alpha_{1}\left(( \alpha _ { 4 } + \alpha _ { 5 } ) \left(\alpha_{2}\left(\alpha_{3}+\alpha_{4}\right)\right.\right. \\
& \left.-\alpha_{1}\left(\alpha_{4}+\mu_{d}\right)\right)-\alpha_{1} \alpha_{4}\left(\mu_{d}+\alpha_{5}\right)+\alpha_{2} \alpha_{5}\left(2 \alpha_{4}+\alpha_{1} r\right)-\alpha_{1} \alpha_{3}\left(\alpha_{4}+3 \alpha_{6}\right. \\
& \left.\left.+\alpha_{7}+2 \mu\right)\right)+\alpha_{1} \alpha_{3} \mu\left(\mu_{d}\left(\alpha_{3}+2 \alpha_{4}+2 \alpha_{6}+\alpha_{7}\right)+\alpha_{4} \alpha_{5}+2\left(\alpha_{3} \alpha_{6}+\alpha_{5}\right.\right. \\
& \left.\left.\left(2 \alpha_{6}+\mu\right)\right)\right)+\alpha_{2} \mu\left(\alpha_{2} r\left(\mu_{d}\left(2 \alpha_{3}+\alpha_{4}+2 \alpha_{5}+2 \mu\right)+\alpha_{5}\left(\alpha_{5}+4 \mu\right)\right)-\alpha_{4}\left(\mu_{d}\right.\right. \\
& \left.\left.\left(\alpha_{4}+4 \alpha_{5}+3 \mu\right)+\alpha_{3}\left(2 \alpha_{5}+\mu\right)\right)-\mu_{d}\left(\alpha_{3}\left(\alpha_{3}+\alpha_{4}+2 \alpha_{5}\right)+\mu_{d}\left(3 \alpha_{5}+2 \mu\right)\right)\right) \\
& \left.+\alpha_{1}\left(\mu_{d} \alpha_{4}\left(B \alpha_{2}+2 \alpha_{5} \mu\right)+\alpha_{6} \mu\left(\mu_{d}\left(\alpha_{7}+4 \mu\right)+4 \alpha_{5} \mu\right)\right)\right\}+\alpha_{1} \alpha_{3} \alpha_{6}\left(\alpha_{1} \alpha_{6} r\right. \\
& \left.\left(\mu_{d} \mu+B \alpha_{2}\right)+2 \alpha_{5} \mu\left(3 \mu_{d} \mu+\alpha_{2} \alpha_{5} r\right)+B \alpha_{2} \alpha_{5}\left(\alpha_{7}+2 \mu_{d}\right)\right)+\alpha_{1} \alpha_{3} \mu\left(\mu_{d} \alpha_{6}{ }^{2}\right. \\
& \left.\left.\left(-2 \alpha_{5}+\alpha_{6}+2 \mu\right)+B \alpha_{1}^{2} \alpha_{5}\left(2 \alpha_{6}-\mu\right)\right)+\alpha_{2} \alpha_{5}^{2} \mu\left(2 \alpha_{2} \alpha_{5} \mu r-\mu_{d} \alpha_{3}^{2}\right)\right] /\left[\alpha_{1}\right. \\
& \left\{\left(\alpha_{1}-\alpha_{2}\right)\left(\mu_{d}^{3}\left(\mu_{d}+2 \mu\right)-\mu_{d} \alpha_{2} r\left(\mu_{d}^{2}+3 \alpha_{5} \mu\right)\right)+\left(\alpha_{3}+\alpha_{4}\right)\left(\mu_{d}^{2}\left(\alpha_{1}-\alpha_{2}\right)\right.\right. \\
& \left.\left(\alpha_{3}-\alpha_{2} r+\mu\right)+2 \mu_{d}^{2}\left(\alpha_{1}-\mu_{d} \alpha_{2}\right)\right)+\left(\alpha_{5}+\alpha_{6}\right)\left(2 \mu_{d}\left(\alpha_{3}+\alpha_{5}\right)\left(\alpha_{1} \alpha_{4}-\alpha_{2} \mu\right)\right. \\
& +\mu_{d}^{2} \alpha_{1}\left(\alpha_{4}+\mu_{d}+2 \mu\right)+\mu_{d}^{2}\left(\alpha_{1}-\alpha_{2}\right)\left(\alpha_{3}+\alpha_{4}+\alpha_{5}+\mu+\mu_{d}\right)-\mu_{d} \alpha_{2}\left(\alpha_{4}\right. \\
& \left.\left.+\mu_{d}+\left(\alpha_{4}+\alpha_{5}\right)\left(\alpha_{3}+\alpha_{5}+\mu_{d}\right)+\alpha_{1} r\left(\alpha_{5}+\mu_{d}\right)\right)-\alpha_{2} \alpha_{4} \alpha_{5}^{2}\right)+\mu_{d}^{2}\left(\alpha_{5}+\mu\right) \\
& \left(\alpha_{2} r\left(3 \alpha_{2}-\alpha_{1}\right)+\alpha_{1}\left(\alpha_{4}+\mu\right)-\alpha_{2} \mu\right)+\left(\alpha_{5}+\alpha_{7}+\mu\right)\left(( \alpha _ { 1 } - \alpha _ { 2 } ) \left(\alpha_{4}^{2}\left(2 \mu_{d}+\alpha_{5}\right)\right.\right. \\
& \left.-2 \mu_{d} \alpha_{2} r\left(\alpha_{3}+\alpha_{4}\right)\right)+\alpha_{2}^{2} \alpha_{5} r\left(\alpha_{3}+\alpha_{4}\right)+\alpha_{2}\left(3 \mu_{d}+\alpha_{5}\right)\left(\alpha_{2} \alpha_{5} r-\alpha_{4} \mu_{d}\right) \\
& \left.-\alpha_{2} \alpha_{4} \alpha_{5}\left(\alpha_{1} r+\mu_{d}\right)\right)+\left(\alpha_{6}+\alpha_{7}+\mu\right)\left(( \alpha _ { 3 } + \alpha _ { 5 } ) \left(2 \mu_{d} \alpha_{1}\left(\alpha_{3}+\mu_{d}\right)+2 \mu_{d}\left(\alpha_{1} \alpha_{6}\right.\right.\right. \\
& \left.\left.-\alpha_{2} \alpha_{3}\right)-\alpha_{2} \alpha_{3} \alpha_{6}\right)+\alpha_{3} \mu_{d}\left(\mu_{d}\left(\alpha_{1}-\alpha_{2}\right)-\alpha_{2}\left(\alpha_{5}+\alpha_{6}\right)\right)+2 \alpha_{1} \alpha_{5}\left(\mu_{d}\left(\alpha_{4}+\mu\right)\right. \\
& \left.+\alpha_{3}\left(\alpha_{4}+\alpha_{6}+\mu\right)\right)+\alpha_{1}\left(\alpha_{3}^{2} \alpha_{6}+2 \mu_{d} \alpha_{2} \mu\right)-\alpha_{2} \alpha_{5}\left(\alpha_{5}\left(2 \mu_{d}+\mu\right)+\alpha_{3}\left(\alpha_{1} r\right.\right. \\
& \left.\left.\left.-\alpha_{4}\right)\right)\right)+\alpha_{1} \mu_{d}\left(\alpha_{6}+\alpha_{7}\right)\left(\alpha_{6} \mu_{d}-\alpha_{2} \alpha_{5} r\right)+\mu_{d}^{2}\left(\alpha_{1}-\alpha_{2}\right)\left(\alpha_{3}+\alpha_{4}\right)\left(\alpha_{4}+\alpha_{5}\right. \\
& +\mu)+\left(\alpha_{6}+\mu\right)\left(\alpha_{5}\left(\alpha_{3}+\alpha_{5}\right)\left(2 \alpha_{1} \mu_{d}-\alpha_{2} \alpha_{5}\right)+\alpha_{1} \alpha_{5}\left(\mu_{d}^{2}-\alpha_{2} \alpha_{5} r+\alpha_{4} \alpha_{5}\right.\right. \\
& \left.\left.+\alpha_{5}\left(\alpha_{4}+\alpha_{6}+\mu\right)\right)\right)-\left(2 \mu_{d}+\alpha_{5}\right)\left(\alpha_{2} \alpha_{5} \mu\left(2 \alpha_{4}+\mu\right)+\mu_{d} \alpha_{2}^{2} \alpha_{7} r\right)+\alpha_{2} \alpha_{3} \alpha_{6}{ }^{2} \\
& \left(\alpha_{1} r-\mu\right)+\mu_{d} \alpha_{5} \mu\left(2 \alpha_{1} \mu-2 \alpha_{2} \alpha_{5}-3 \mu_{d}\right)-\mu_{d}^{2} \alpha_{5}\left(2 \alpha_{5}+\alpha_{1} \mu\right)+\mu_{d} \alpha_{1} \mu \\
& \left(2 \alpha_{3} \alpha_{6}+\alpha_{4}\left(4 \alpha_{5}-\mu_{d}\right)\right)+\alpha_{2}^{2} \alpha_{5}^{2} \mu r+\left(\alpha_{7}+\mu\right)\left(( \alpha _ { 7 } + \mu ) \left(( \alpha _ { 1 } - \alpha _ { 2 } ) \left(-\alpha_{2} r\right.\right.\right.
\end{aligned}
$$




$$
\begin{aligned}
& \left.\left(\mu_{d}+\mu\right)+\mu_{d} \alpha_{6}+\mu^{2}\right)+\alpha_{1}\left(\alpha_{6}+\mu\right)\left(2 \alpha_{4}+\alpha_{6}\right)-\alpha_{2}\left(\alpha_{4}\left(\alpha_{5}+\mu\right)+\alpha_{6}\left(\alpha_{3}+\alpha_{4}\right)\right. \\
& \left.\left.+\mu\left(\alpha_{4}+\alpha_{6}\right)\right)+\alpha_{1}\left(\alpha_{6}\left(\mu_{d}+\mu\right)-\alpha_{2} r\left(\alpha_{3}+\alpha_{6}\right)+2 \mu_{d}\left(\alpha_{3}+\alpha_{4}+\mu\right)\right)\right)+\left(\alpha_{5}\right. \\
& \left.+\alpha_{7}+\mu\right)\left(\alpha_{4}\left(\alpha_{1}-\alpha_{2}\right)\left(\alpha_{4}-\alpha_{2} r\right)+\alpha_{2}\left(\alpha_{2} r\left(\alpha_{3}+\alpha_{5}\right)-2 \mu_{d} \alpha_{4}+\alpha_{5} \alpha_{6}\right)\right)+\left(\alpha_{6}\right. \\
& \left.+\alpha_{7}+\mu\right)\left(\mu_{d}\left(\mu_{d}\left(\alpha_{1}-\alpha_{2}\right)-\alpha_{2}\left(2 \alpha_{3}+\alpha_{5}+2 \mu\right)\right)+\alpha_{3}\left(\alpha _ { 1 } \left(\alpha_{3}+2 \alpha_{4}+2 \alpha_{6}\right.\right.\right. \\
& \left.\left.+2 \mu)-\alpha_{2}\left(\alpha_{3}+2 \alpha_{4}+2 \alpha_{5}+2 \mu\right)\right)-\alpha_{2} \mu\left(2 \alpha_{3}+\alpha_{5}\right)\right)+\left(\alpha_{6}+\mu\right)\left(\alpha _ { 5 } \left(\mu \left(2 \alpha_{1}\right.\right.\right. \\
& \left.\left.-\alpha_{2}\right)-\alpha_{2}\left(2 \alpha_{3}+\alpha_{5}+2 \alpha_{1} r+2 \mu_{d}\right)+2 \alpha_{1}\left(2 \alpha_{4}+\alpha_{6}\right)\right)+\mu_{d}^{2}\left(\alpha_{1}-\alpha_{2}\right)-\mu_{d} \alpha_{2} \\
& \left.\left(\alpha_{3}+\alpha_{5}\right)\right)-\mu_{d} \mu\left(\alpha_{1}-\alpha_{2}\right)\left(\alpha_{2} r-2 \mu\right)+3 \mu_{d}\left(\alpha_{3}+\mu\right)\left(\alpha_{1} \mu_{d}-\alpha_{2} \alpha_{3}\right)-2 \mu_{d} \\
& \left(\alpha_{2} \mu_{d}\left(\alpha_{3}+2 \alpha_{5}\right)-2 \alpha_{1} \mu\left(\alpha_{3}+\alpha_{4}+\alpha_{6}\right)\right)-3 \mu_{d} \alpha_{2} \alpha_{5} \mu-\alpha_{2} \mu\left(\alpha_{5}\left(\mu-2 \alpha_{2} r\right)\right. \\
& \left.+4 \alpha_{4}\left(\mu_{d}+\alpha_{5}\right)\right)-\mu_{d}^{2}\left(3 \alpha_{2} \mu-\alpha_{1} \alpha_{6}\right)+\alpha_{1} \mu_{d}\left(2 \alpha_{4} \alpha_{6}-\alpha_{2} r\left(2 \mu_{d}+\alpha_{5}+2 \alpha_{6}\right)\right) \\
& +2 \mu_{d}^{2}\left(\alpha_{1} \mu_{d}-\alpha_{2}\right)+\mu_{d}^{2}\left(\alpha_{1}-\alpha_{2}\right)\left(\alpha_{3}+\alpha_{4}\right)+\alpha_{3} \mu_{d}\left(4 \alpha_{1}\left(\alpha_{4}+\alpha_{6}\right)-\alpha_{2}\left(\alpha_{4}\right.\right. \\
& \left.\left.\left.\left.\left.+\alpha_{5}\right)\right)+\left(\alpha_{5}+\alpha_{6}\right)\left(2 \mu_{d} \alpha_{1}\left(\alpha_{4}+\alpha_{6}\right)-2 \alpha_{2} \alpha_{5}\left(\alpha_{4}+\mu_{d}\right)\right)\right)\right\}\right]
\end{aligned}
$$

and

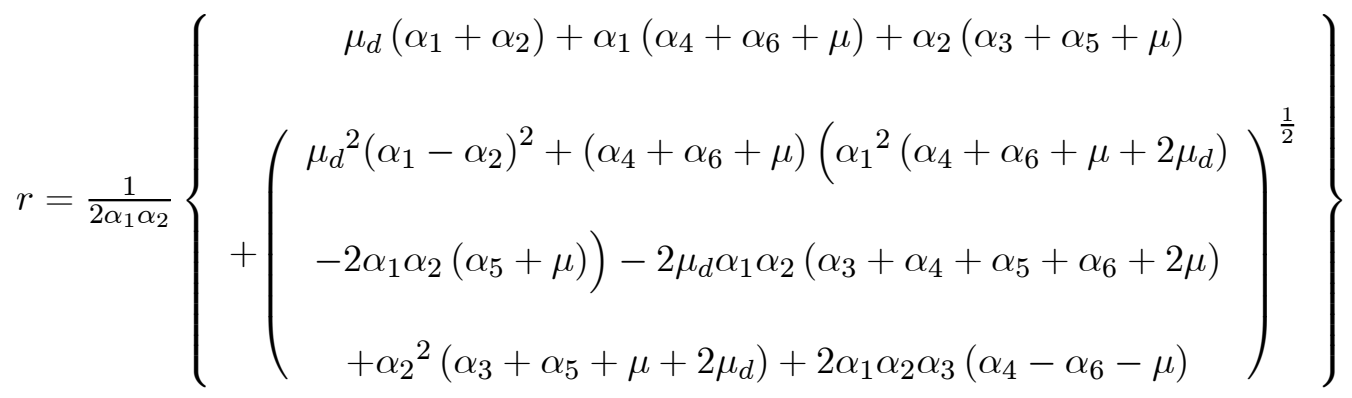

Now, we evaluate the basic reproduction number known as threshold $R_{0}$ using next generation matrix method [6].

$$
\text { Let } X^{\prime}=\left(S_{M}, I_{1}, I_{2}, M\right)^{\prime} \text { and } X^{\prime}=\frac{d X}{d t}=F(X)-V(X)
$$

where, $F(X)$ denotes the rate of arrival of new individual in the compartment and $V(X)$ denotes the rate of transmission of infection which are given by

$F(X)=\left[\begin{array}{l}\alpha_{1} S_{M} I_{1} \\ \alpha_{2} S_{M} I_{2} \\ 0 \\ 0\end{array}\right]$ and $V(X)=\left[\begin{array}{l}\alpha_{3} I_{1}-\alpha_{4} I_{2}+\alpha_{5} I_{1}+\left(\mu+\mu_{d}\right) I_{1} \\ -\alpha_{3} I_{1}+\alpha_{4} I_{2}+\alpha_{6} I_{2}+\left(\mu+\mu_{d}\right) I_{2} \\ -\alpha_{5} I_{1}-\alpha_{6} I_{2}+\alpha_{7} M+\left(\mu+\mu_{d}\right) M \\ -B+\alpha_{1} S_{M} I_{1}+\alpha_{2} S_{M} I_{2}-\alpha_{7} M+\mu S_{M}\end{array}\right]$ Now, $D F\left(X_{0}\right)=\left[\begin{array}{ll}f & 0 \\ 0 & 0\end{array}\right]$ and $D V\left(X_{0}\right)=\left[\begin{array}{ll}v & 0 \\ J_{1} & J_{2}\end{array}\right]$, where $f$ and $v$ are $4 \times 4$ matrices defined as $f=\left[\frac{\partial F_{i}\left(X_{0}\right)}{\partial X_{j}}\right]$ and $v=\left[\frac{\partial V_{i}\left(X_{0}\right)}{\partial X_{j}}\right]$ 
Finding $f$ and $v$, we get

$$
\begin{gathered}
f=\left[\begin{array}{llll}
\alpha_{1} S_{M} & 0 & 0 & \alpha_{1} I_{1} \\
0 & \alpha_{2} S_{M} & 0 & \alpha_{2} I_{2} \\
0 & 0 & 0 & 0 \\
0 & 0 & 0 & 0
\end{array}\right] \text { and } \\
v=\left[\begin{array}{llll}
\alpha_{3}+\alpha_{5}+\mu+\mu_{d} & -\alpha_{4} & 0 & \\
-\alpha_{3} & \alpha_{4}+\alpha_{6}+\mu+\mu_{d} & 0 & 0 \\
-\alpha_{5} & -\alpha_{6} & \alpha_{7}+\mu+\mu_{d} & 0 \\
\alpha_{1} S_{M} & \alpha_{2} S_{M} & -\alpha_{7} & \alpha_{1} I_{1}+\alpha_{2} I_{2}+\mu
\end{array}\right]
\end{gathered}
$$

Here, $v$ is non-singular matrix.

For this model, matrix $f v^{-1}$ is calculated and the basic reproduction (threshold) $R_{0}$ at $Y_{0}$ is obtained as

$$
R_{0}=\frac{B\left(\alpha_{1} \alpha_{4}+\alpha_{2} \alpha_{3}\right)}{\mu\left(\left(\mu+\mu_{d}\right)^{2}+\left(\alpha_{3}+\alpha_{4}+\alpha_{5}+\alpha_{6}\right)\left(\mu+\mu_{d}\right)+\alpha_{3} \alpha_{6}+\alpha_{5}\left(\alpha_{4}+\alpha_{6}\right)\right)}
$$

\section{Stability}

\subsection{Local Stability}

In this section, for both equilibria points local as well as global stability is discussed.

Theorem 3.1.1 (Stability at $\left.Y_{0}\right): Y_{0}=\left(\frac{B}{\mu}, 0,0,0\right)$ is locally asymptotically stable if $\mu\left(\left(\alpha_{4}+\alpha_{6}\right)\left(B \alpha_{1}-\alpha_{5} \mu\right)+B \alpha_{2}\left(\alpha_{3}+\alpha_{5}\right)\right)<$ $\left(\mu\left(\mu_{d}+\mu\right)\left(\mu\left(\alpha_{3}+\alpha_{4}+\alpha_{5}+\alpha_{6}+\mu\right)-\left(\alpha_{1}+\alpha_{2}\right) B\right)+B^{2} \alpha_{1} \alpha_{2}+\mu^{2} \alpha_{3} \alpha_{6}\right)$.

Proof: The Jacobian matrix $J_{0}$ at $Y_{0}$ is given by 


$$
J_{0}=\left[\begin{array}{llll}
-\mu & -\frac{\alpha_{1} B}{\mu} & -\frac{\alpha_{2} B}{\mu} & \alpha_{7} \\
0 & \frac{\alpha_{1} B}{\mu}-\mu_{d}-\alpha_{3}-\alpha_{5}-\mu & \alpha_{4} & 0 \\
0 & \alpha_{3} & \frac{\alpha_{2} B}{\mu}-\mu_{d}-\alpha_{4}-\alpha_{6}-\mu & 0 \\
0 & \alpha_{5} & \alpha_{6} & -\alpha_{7}-\mu-\mu_{d}
\end{array}\right]
$$

Jacobian $J_{0}$ has eigenvalues,

$$
\begin{aligned}
& \lambda_{1}=-\mu<0, \lambda_{2}=-\left(\alpha_{7}+\mu+\mu_{d}\right)<0 \\
& \lambda_{3}=\frac{1}{\mu}\left(\begin{array}{c}
-\frac{\mu}{2}\left(\alpha_{2}+\alpha_{3}+\alpha_{4}+\alpha_{5}+\alpha_{6}+2\left(\mu+\mu_{d}\right)\right)+\frac{B}{2}\left(\alpha_{1}+\alpha_{2}\right) \\
+\frac{1}{2}\left(\begin{array}{c}
B^{2}\left(\alpha_{1}-\alpha_{2}\right)^{2}-2 B \mu\left(\alpha_{1}-\alpha_{2}\right)\left(\alpha_{3}-\alpha_{4}+\alpha_{5}-\alpha_{6}\right) \\
+\mu^{2}\left(\left(\alpha_{3}+\alpha_{4}\right)^{2}+2\left(\alpha_{5}-\alpha_{6}\right)\left(\alpha_{3}-\alpha_{4}\right)+\left(\alpha_{5}-\alpha_{6}\right)^{2}\right)
\end{array}\right)
\end{array}\right)^{\frac{1}{2}} \lambda_{4}=\frac{1}{\mu}\left(\begin{array}{c}
-\frac{\mu}{2}\left(\alpha_{2}+\alpha_{3}+\alpha_{4}+\alpha_{5}+\alpha_{6}+2\left(\mu+\mu_{d}\right)\right)+\frac{B}{2}\left(\alpha_{1}+\alpha_{2}\right) \\
-\frac{1}{2}\left(\begin{array}{c}
B^{2}\left(\alpha_{1}-\alpha_{2}\right)^{2}-2 B \mu\left(\alpha_{1}-\alpha_{2}\right)\left(\alpha_{3}-\alpha_{4}+\alpha_{5}-\alpha_{6}\right) \\
+\mu^{2}\left(\left(\alpha_{3}+\alpha_{4}\right)^{2}+2\left(\alpha_{5}-\alpha_{6}\right)\left(\alpha_{3}-\alpha_{4}\right)+\left(\alpha_{5}-\alpha_{6}\right)^{2}\right)
\end{array}\right)^{\frac{1}{2}}
\end{array}\right)
\end{aligned}
$$

Here $\lambda_{3}$ and $\lambda_{4}$ are negative if

$\mu\left(\left(\alpha_{4}+\alpha_{6}\right)\left(B \alpha_{1}-\alpha_{5} \mu\right)+B \alpha_{2}\left(\alpha_{3}+\alpha_{5}\right)\right)<$

$\left(\mu\left(\mu_{d}+\mu\right)\left(\mu\left(\alpha_{3}+\alpha_{4}+\alpha_{5}+\alpha_{6}+\mu\right)-\left(\alpha_{1}+\alpha_{2}\right) B\right)+B^{2} \alpha_{1} \alpha_{2}+\mu^{2} \alpha_{3} \alpha_{6}\right)$

As all eigenvalues of matrix are negative, equilibrium point of system is stable.

Theorem 3.1.2 (Stability at $Y^{*}$ ): Endemic point $Y^{*}$ is locally asymptotically stable if the following conditions hold:

i) $x_{5}$ and $x_{7}<0$,

ii) $\alpha_{3}\left(x_{1}+x_{8}\right)<x_{3} x_{4}$,

iii) $x_{4}\left(x_{7}+x_{8}\right)>\alpha_{5} \alpha_{7}$,

iv) $x_{6}\left(x_{5}+x_{8}\right)>\alpha_{6} \alpha_{7}$, v) $x_{2} x_{6}<\alpha_{4} x_{1}$, vi) $\alpha_{5} x_{7}>\alpha_{3} \alpha_{6}$, vii) $\alpha_{6} x_{5}>$ $\alpha_{4} \alpha_{5}$

Proof: We determine the local stability behavior of endemic equilibrium point $Y^{*}$ by using the Jacobian matrix $J^{*}$ which is given by 


$$
J^{*}=\left[\begin{array}{llll}
x_{1} & x_{4} & x_{6} & \alpha_{7} \\
x_{2} & x_{5} & \alpha_{4} & 0 \\
x_{3} & \alpha_{3} & x_{7} & 0 \\
0 & \alpha_{5} & \alpha_{6} & x_{8}
\end{array}\right]
$$

where, $x_{1}=-\left(\alpha_{1} I_{1}{ }^{*}+\alpha_{2} I_{2}{ }^{*}+\mu\right), x_{2}=\alpha_{1} I_{1}{ }^{*}, x_{3}=\alpha_{2} I_{2}{ }^{*}, x_{4}=-\alpha_{1} S_{M}{ }^{*}$,

$$
\begin{aligned}
& x_{5}=\alpha_{1} S_{M}{ }^{*}-\alpha_{3}-\alpha_{5}-\mu-\mu_{d}, x_{6}=-\alpha_{1} S_{M}{ }^{*}, \\
& x_{7}=\alpha_{2} S_{M}{ }^{*}-\alpha_{4}-\alpha_{6}-\mu-\mu_{d}, x_{8}=-\left(\alpha_{7}+\mu+\mu_{d}\right)
\end{aligned}
$$

The corresponding characteristic equation of Jacobian matrix is

$$
\lambda^{4}+a_{1} \lambda^{3}+a_{2} \lambda^{2}+a_{3} \lambda+a_{4}=0
$$

where,

$$
\begin{aligned}
& a_{1}=-x_{1}-x_{5}-x_{7}-x_{8} \\
& a_{2}=x_{1}\left(x_{5}+x_{7}+x_{8}\right)+x_{5}\left(x_{7}+x_{8}\right)+x_{7} x_{8}-\alpha_{3} \alpha_{4}-x_{2} x_{4}-x_{3} x_{6} \\
& a_{3}=\alpha_{4}\left(\alpha_{3}\left(x_{1}+x_{8}\right)-x_{3} x_{4}\right)+x_{2}\left(x_{4}\left(x_{7}+x_{8}\right)-\alpha_{5} \alpha_{7}\right)+x_{3}\left(x_{6}\left(x_{5}+x_{8}\right)\right. \\
& \left.\quad-\alpha_{6} \alpha_{7}\right)+\alpha_{3} x_{8}\left(x_{2} x_{6}-\alpha_{4} x_{1}\right)+\alpha_{7} x_{2}\left(\alpha_{5} x_{7}-\alpha_{3} \alpha_{6}\right)-x_{1}\left(x_{5}\left(x_{7}+x_{8}\right)\right. \\
& \left.\quad+x_{7} x_{8}\right)-x_{5} x_{7} x_{8}-\alpha_{3} x_{2} x_{6}, \\
& a_{4}=\alpha_{3} x_{8}\left(x_{2} x_{6}-\alpha_{4} x_{1}\right)+\alpha_{7} x_{2}\left(\alpha_{5} x_{7}-\alpha_{3} \alpha_{6}\right)+\alpha_{7} x_{3}\left(\alpha_{6} x_{5}-\alpha_{4} \alpha_{5}\right) \\
& \quad+x_{8}\left(x_{4}\left(\alpha_{4} x_{3}-x_{2} x_{7}\right)+x_{5}\left(x_{1} x_{7}-x_{3} x_{6}\right)\right)
\end{aligned}
$$

Here, $x_{1}, x_{4}, x_{6}, x_{8}<0$ and $x_{2}, x_{3}>0$ is obvious.

The equilibrium point is locally asymptotically stable [15], if following conditions are hold:

i) $x_{5}$ and $x_{7}<0$,

ii) $\alpha_{3}\left(x_{1}+x_{8}\right)<x_{3} x_{4}$,

iii) $x_{4}\left(x_{7}+x_{8}\right)>\alpha_{5} \alpha_{7}$,

iv) $x_{6}\left(x_{5}+x_{8}\right)>\alpha_{6} \alpha_{7}$, v) $x_{2} x_{6}<\alpha_{4} x_{1}$, vi) $\alpha_{5} x_{7}>\alpha_{3} \alpha_{6}$, vii) $\alpha_{6} x_{5}>$ $\alpha_{4} \alpha_{5}$

\subsection{Global Stability}

Now, let us discuss the global stability behavior of the equilibrium $Y_{0}$ and $Y^{*}$ using Lyapunov's function. 
Theorem 3.2.1 (Stability at $Y_{0}$ ): The disease-free equilibrium point $Y_{0}$ is globally asymptotically stable.

Proof: Consider the Lyapunov's function

$$
L_{1}(t)=I_{1}(t)+I_{2}(t)+M(t)
$$

then

$$
\begin{aligned}
L_{1}{ }^{\prime}(t) & =I_{1}{ }^{\prime}(t)+I_{2}{ }^{\prime}(t)+M^{\prime}(t) \\
& =S_{M}\left(\alpha_{1} I_{1}+\alpha_{2} I_{2}\right)-\left(I_{1}+I_{2}+M\right)\left(\mu+\mu_{d}\right)-\alpha_{7} M
\end{aligned}
$$

we get $\frac{d L_{1}}{d t}<0$ if $\alpha_{1} B<\mu^{2}$ and $\alpha_{2} B<\mu^{2}$

whereas, $\frac{d L_{1}}{d t}=0$ only if $I_{1}, I_{2}, M=0$.

Therefore, $Y_{0}$ is globally asymptotically stable [11].

Theorem 3.2.2 (Stability at $Y^{*}$ ): The endemic equilibrium point $Y^{*}$ is globally asymptotically stable.

Proof: Consider the Lyapunov function,

$L(t)=\frac{1}{2}\left[\left(S_{M}-S_{M^{*}}\right)+\left(I_{1}-I_{1}{ }^{*}\right)+\left(I_{2}-I_{2}{ }^{*}\right)+\left(M-M^{*}\right)\right]^{2}$

then

$$
\begin{aligned}
L^{\prime}(t)= & {\left[\left(S_{M}-S_{M}{ }^{*}\right)+\left(I_{1}-I_{1}{ }^{*}\right)+\left(I_{2}-I_{2}{ }^{*}\right)\right.} \\
& \left.+\left(M-M^{*}\right)\right]\left(S_{M}{ }^{\prime}+I_{1}{ }^{\prime}+I_{2}{ }^{\prime}+M^{\prime}\right) \\
& =-\left[\left(\left(S_{M}-S_{M}{ }^{*}\right)+\left(I_{1}-I_{1}{ }^{*}\right)+\left(I_{2}-I_{2}{ }^{*}\right)+\left(M-M^{*}\right)\right)\right. \\
& \left(\mu\left(\left(S_{M}-S_{M}{ }^{*}\right)+\left(I_{1}-I_{1}{ }^{*}\right)+\left(I_{2}-I_{2}{ }^{*}\right)+\left(M-M^{*}\right)\right)\right. \\
& \left.\left.+\mu_{d}\left(I_{1}+I_{2}+M\right)\right)\right]<0
\end{aligned}
$$

Here, we denote $B=\mu\left(S_{M}{ }^{*}+I_{1}{ }^{*}+I_{2}{ }^{*}+M^{*}\right)$.

Therefore, $Y^{*}$ is globally stable.

In next section, we validate the model by numerical simulation using the parametric values given in Table 1 and system of equation (2.1). 


\section{Numerical Simulations}

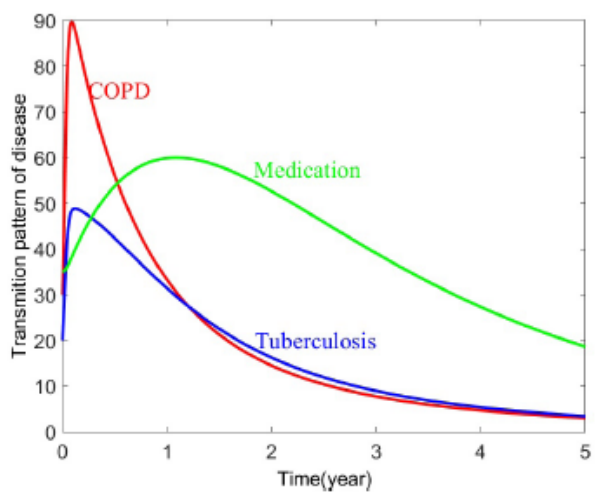

Figure 2: Transmission pattern of diseased individuals procuring medication

Figure 2 shows the transmission pattern of the disease. It can be noted that as the disease is detected, around 35 individuals starts taking precautions or medication. Some may neglect the symptoms, but after approximately a year they starts procuring medications. Also, around 29 COPD patients suffer from tuberculosis after a year.

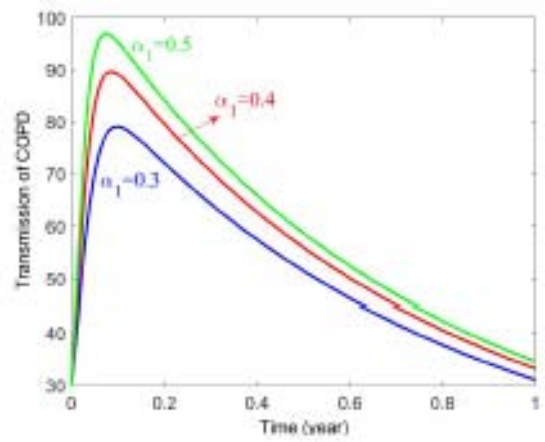

Figure 3: Effect of change in $\alpha_{1}$ on COPD
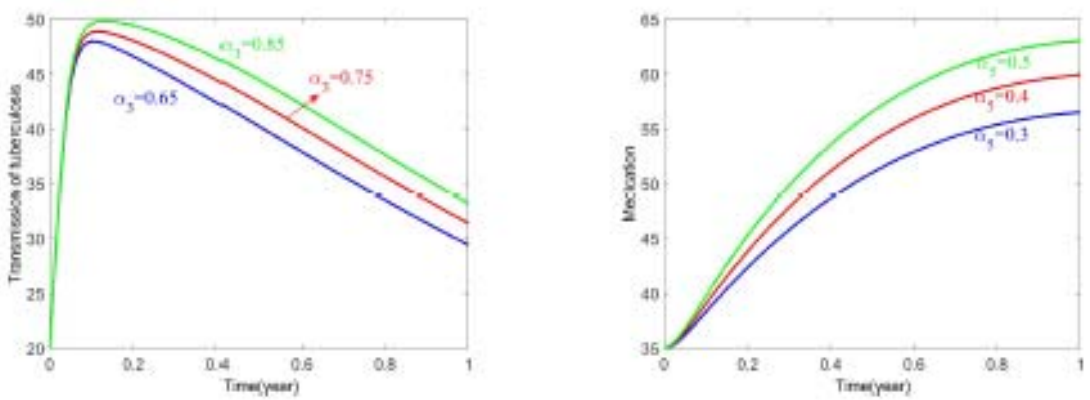

Figure 4: Effect of change in $\alpha_{3}$ on Figure 5: Impact of change of $\alpha_{5}$ tuberculosis on medication 
Increase in the rates corresponding to disease and medication results in change of outputs. So, the following graphs is to justify the findings. As shown in Figure 3, increase in $\alpha_{1}$ by $20 \%$ results in increase of individual suffering from COPD by $20.79 \%$. Also, $4.07 \%$ increase of individual suffering from tuberculosis will be observed if $\alpha_{3}$ increases by 20\% (Figure 4 ). Figure 5 shows that $20 \%$ increase in rate of individuals suffering from COPD $\left(\alpha_{5}\right)$ results in increase of rate of individual procuring medication from 56 individuals to 63 individuals.
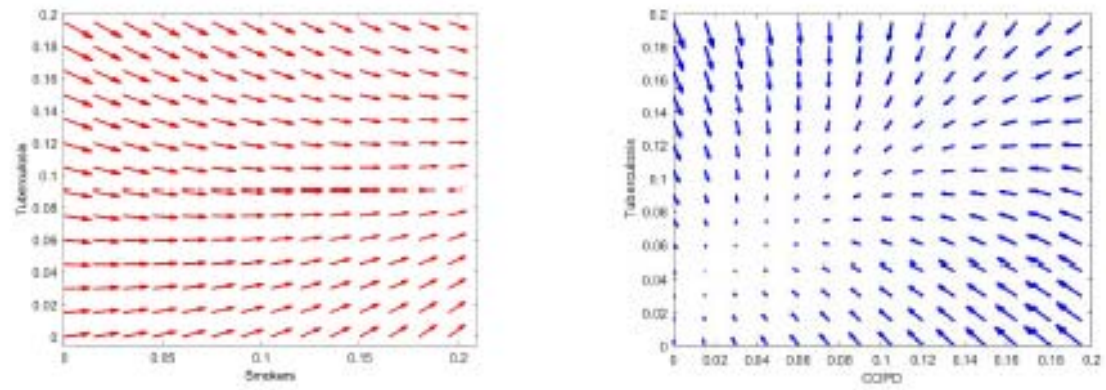

Figure 6: Intensity of tuberculosis Figure 7: Directional flow of among smokers

COPD and tuberculosis
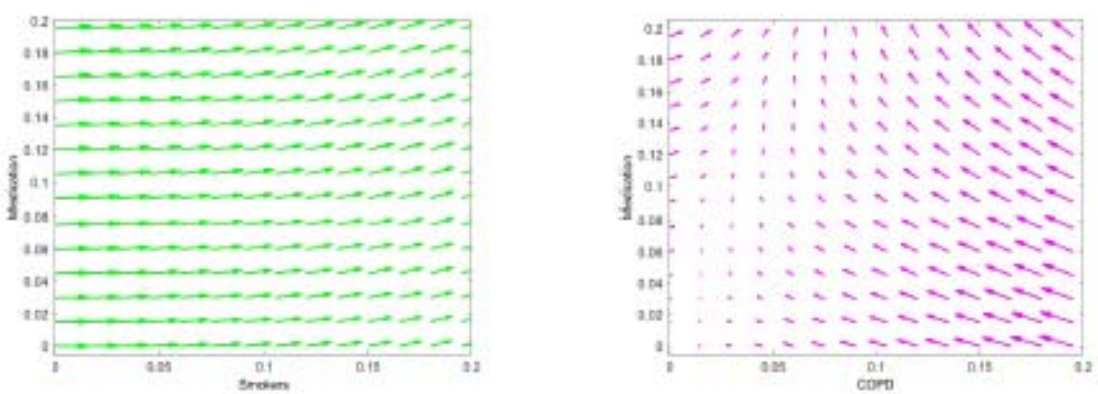

Figure 8: Intensity of medication Figure 9: Intensity flow of COPD among smokers towards medication 
To visualize the intensity or flow among the compartments, following quiver graphs are analyzed. Intensity of individuals suffering from tuberculosis due to smoking gradually decreases after taking proper medication (Figure 6). Intensity of individuals suffering from COPD is more as compared to tuberculosis, but it gradually decreases after taking proper medication (Figure 7). Intensity of medication increases among smokers and slowly decreases as they start getting cured or quit smoking (Figure 8). Intensity of individuals suffering from COPD decreases while taking medication (Figure $9)$.

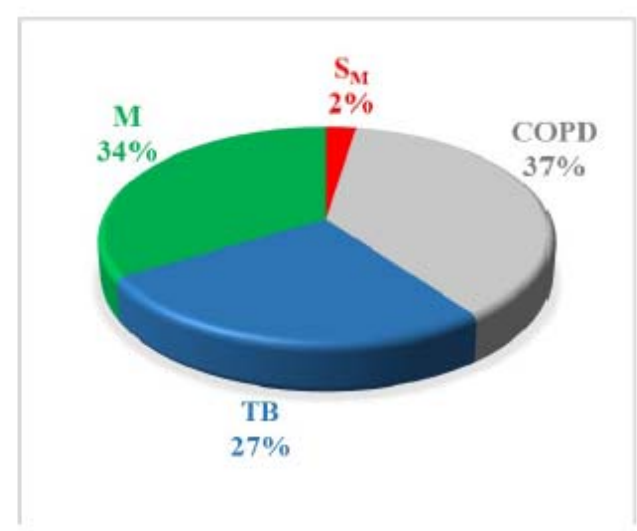

Figure 10: Percentage of all compartment

Here, among $2 \%$ smokers, $37 \%$ are affected by COPD and $27 \%$ by Tuberculosis. Out of these many patients, $34 \%$ procures medication (Figure 10).

\section{Conclusion}

To study the impact of medication on individuals suffering from COPD and tuberculosis due to smoking, a mathematical model is formulated. Equilibrium points are calculated for proposed system of nonlinear ordinary differential equations. Also, at both equilibrium points, system is locally as well as globally stable. A basic reproduction number has been evaluated which is equal to 0.7064 which connotes that approximately $71 \%$ of the individuals in the society are suffering from COPD and tuberculosis due to smoking who needs medication. Numerical simulations have been carried out to justify the results, which interprets that with the increase in the rate of affected individuals due to smoking will increases the morbidity and mortality rate all over the world. Also, quiver graph shows the intensity 
or flow of individuals in their respective compartment. To keep the body healthy and protect lungs and other symptoms from getting worse, smoking cessation is the best thing one can do. This can help people to stay more active and enjoy life.

\section{Acknowledgement}

The authors thank reviewers for their constructive comments and DSTFIST file \# MSI-097 for technical support to the department. 4th author (Ankush H. Suthar) is founded by a Junior Research Fellowship from the Council of Scientific and Industrial Research (file no. 09/070(0061)/2019EMR-I).

\section{References}

[1] M. Adak, "Effects of smoking and need for cessation: biochemical and pharmacological feedback", Biochemistry \& pharmacology, vol. 03, no. 05, p. 1000145, 2014, doi: 10.4172/2167-0501.1000145.

[2] M. Al-Hamdani, D. B. Hopkins, and T. Park, "Vaping among youth and young adults: a 'red alert' state", Journal of public health policy, vol. 41, no. 1, pp. 63-69, Oct. 2019, doi: 10.1057/s41271-019-00193-2.

[3] A. S. Buist, M. A. Mcburnie, W. M. Vollmer, S. Gillespie, P. Burney, D. M. Mannino, A. M. Menezes, S. D. Sullivan, T. A. Lee, K. B. Weiss, R. L. Jensen, G. B. Marks, A. Gulsvik, and E. Nizankowska-Mogilnicka, "International variation in the prevalence of COPD (The BOLD Study): a population-based prevalence study", The Lancet, vol. 370, no. 9589, pp. 741-750, Sep. 2007, doi: 10.1016/S01406736(07)61377-4.

[4] D. Dabas, "Therapies for smoking cessation: a meta-analysis of double-blind randomized controlled trials", Journal of health sciences \& research, vol. 9, no. 2, pp. 49-56, 2018., doi: 10.5005/jp-journals-10042-1069.

[5] P. Davies, W. W. Yew, D. Ganguly, A. L. Davidow, L. B. Reichman, K. Dheda, and G. A. Rook, "Smoking and tuberculosis: the epidemiological association and immunopathogenesis", Transactions of the Royal Society of Tropical Medicine and Hygiene, vol. 100, no. 4, pp. 291-298, Apr. 2006, doi: 10.1016/j.trstmh.2005.06.034.

[6] O. Diekmann, J. A. P. Heesterbeek, and M. G. Roberts, "The construction of nextgeneration matrices for compartmental epidemic models", Journal of The Royal Society Interface, vol. 7, no. 47, pp. 873-885, Nov. 2009, doi: 10.1098/rsif.2009.0386.

[7] J.-Y. Feng, S.-F. Huang, W.-Y. Ting, M.-C. Lee, Y.-C. Chen, Y.-Y. Lin, Y.-C. Lee, and W.-J. Su, "Impact of cigarette smoking on latent tuberculosis infection: does age matter?", European respiratory journal, vol. 43, no. 2, pp. 630-632, Sep. 2013., doi: 10.1183/09031936.00118313. 
[8] R. Gupta, A. Bharat, U. Dhiman, and A. Sharma, "Nicotine replacement therapy: A smoking cessation aid... an overview", International journal of oral health dentistry, vol. 5, no. 2, pp. 69-75, Jul. 2019, doi: 10.18231/j.ijohd.2019.017.

[9] P. Jha, B. Jacob, V. Gajalakshmi, P. C. Gupta, N. Dhingra, R. Kumar, D. N. Sinha, R. P. Dikshit, D. K. Parida, R. Kamadod, J. Boreham, and R. Peto, "A nationally representative case-control study of smoking and death in India", New England journal of medicine, vol. 358, no. 11, pp. 1137-1147, Mar. 2008, doi: 10.1056/NEJMsa0707719.

[10] S. Tamgadge, "Tobacco cessation - scissor the consumption - a review", International clinical pathology journal, vol. 5, no. 3, pp. 241-246, Nov. 2017, doi: 10.15406/icpjl.2017.05.00131.

[11] J. P. LaSalle, The stability of dynamical systems, vol. 25. Philadelphia, PA: SIAM, 1976, doi: $10.1137 / 1.9781611970432$.

[12] C.-H. Lee, M.-C. Lee, C.-C. Shu, C.-S. Lim, J.-Y. Wang, L.-N. Lee, and K.-M. Chao, "Risk factors for pulmonary tuberculosis in patients with chronic obstructive airway disease in Taiwan: a nationwide cohort study", BMC infectious diseases, vol. 13, no. 1, art. ID. 194, Apr. 2013, doi: 10.1186/1471-2334-13-194.

[13] C. C. Leung, W. W. Yew, C. K. Chan, K. C. Chang, W. S. Law, S. N. Lee, L. B. Tai, E. C. Leung, R. K. Au, S. S. Huang, and C. M. Tam, "Smoking adversely affects treatment response, outcome and relapse in tuberculosis", European respiratory journal, vol. 45 , no. 3 , pp. 738-745, Apr. 2014, doi: 10.1183/09031936.00114214.

[14] H.-H. Lin, M. Murray, T. Cohen, C. Colijn, and M. Ezzati, "Effects of smoking and solid-fuel use on COPD, lung cancer, and tuberculosis in China: a time-based, multiple risk factor, modelling study", The Lancet, vol. 372, no. 9648, pp. 1473-1483, Oct. 2008, doi: 10.1016/S0140-6736(08)61345-8.

[15] E. J. Routh, A treatise on the stability of a given state of motion: particularly steady motio. London: Macmillan and Co., 1877. [On line]. Available: https://bit.ly/2Yh90GC

[16] S. S. Salvi and P. J. Barnes, "Chronic obstructive pulmonary disease in nonsmokers," The Lancet, vol. 374, no. 9691, pp. 733-743, Aug. 2009, doi: 10.1016/S0140-6736(09)61303-9.

[17] R. N. V. Z. Smit, M. Pai, W. W. Yew, C. C. Leung, A. Zumla, E. D. Bateman, and K. Dheda, "Global lung health: the colliding epidemics of tuberculosis, tobacco smoking, HIV and COPD", European respiratory journal, vol. 35, no. 1, pp. 27-33, Dec. 2009, doi: 10.1183/09031936.00072909.

[18] S. Widodo, R. N. Rohmah, B. Handaga, and L. D. D. Arini, "Lung diseases detection caused by smoking using support vector machine", TELKOMNIKA (Telecommunication computing electronics and control), vol. 17, no. 3, pp. 1256-1266, Jun. 2019, doi: 10.12928/telkomnika.v17i3.9799.

[19] C. Zaher, R. Halbert, R. Dubois, D. George, and D. Nonikov, "Smoking-related diseases: the importance of COPD", The international journal of tuberculosis and lung disease, vol. 8, no. 12, pp. 1423-1428, Dec. 2004. [On line]. Available: https://bit.ly/3eSS9zT 